\title{
A Study of Chirality in Gold Nanostructures with Second Harmonic Generation
}

\author{
V.K. ValeV ${ }^{a}, *$ A.V. SilhaneK ${ }^{b}$, W. Gillijns ${ }^{b}$, V.V. Moshchalkov ${ }^{b}$ \\ AND T. VERBIEST ${ }^{a}$ \\ ${ }^{a}$ Molecular Electronics and Photonics Group, INPAC, Katholieke Universiteit Leuven \\ Celestijnenlaan 200D, B-3001, Leuven, Belgium \\ ${ }^{b}$ Nanoscale Superconductivity and Magnetism, Pulsed Fields Group \\ INPAC, Institute Katholieke Universiteit Leuven, Belgium
}

\begin{abstract}
We present the second harmonic generation - circular dichroism response from G-shaped gold nanostructures for $P$-polarized incoming light. By eliminating the contributions of anisotropy to the signal, an absolute measure of the second harmonic generation - circular dichroism is presented. The system of planar nanostructures is an excellent model for studying the correlation between optical properties and chiral morphology at the nanoscale. Samples with both enantiomorphs of the chiral pattern were prepared and, demonstrating the unambiguous presence of chirality, the second harmonic generation - circular dichroism response from two such samples was fitted simultaneously with a single set of fitting parameters.
\end{abstract}

PACS numbers: 42.65.Ky, 81.05.Xj, 78.67.Pt, 36.20.Ey

\section{Introduction}

Numerous biomolecules are said to be chiral, i.e. they are shaped with a sense of "twist". Chirality is the geometric property of rigid objects, or groups of atoms, of being non-superimposable on their mirror image. The two geometrical structures are then distinguishable by their handedness and are designated as "enantiomorphs". Experimental techniques capable of distinguishing between enantiomorphs are highly desired in the fields of biology, chemistry, pharmacology and medicine.

Second harmonic generation - circular dichroism (SHG-CD) [1, 2] is a nonlinear optical technique with demonstrated sensitivity at the molecular level for both surfaces and interfaces. The method is well known for being capable of distinguishing between left or right handed molecules, with a sensitivity that is much greater than that of linear probes, especially in systems involving monolayers of organic molecules. Organic molecules, however, can be difficult to synthesize and arrange at the monolayer level. Therefore, patterned metallic thin films, in which chirality can be tailored according to specifications, would constitute a very useful model system for studying the dependence of SHG-CD on the degree of chirality.

\section{Experimental part}

The investigated samples consist of a periodic array of G-shaped microstructures made of a $\mathrm{Au}(25 \mathrm{~nm})$

\footnotetext{
* corresponding author; e-mail: v.k.valev@fys.kuleuven.be
}

film evaporated by DC sputtering system on the top of a $\mathrm{Si} / \mathrm{SiO}_{2}$ substrate. For the sample preparation, we first cover the substrate with a double polymethyl metacrylate-methyl metacrylate resist layer in which the array of G-shaped structures is defined by electron-beam lithography. After the bilayer deposition, the resist is removed by a liftoff procedure. Figure 1 shows scanning electron microscopy images of the two chiral structures investigated. The lateral size of each individual motif is $1 \mu \mathrm{m}$ wide, the line width is $200 \mathrm{~nm}$ and the separation between neighboring structures is $200 \mathrm{~nm}$. The whole array covers an area of $2.5 \times 2.5 \mathrm{~mm}^{2}$.

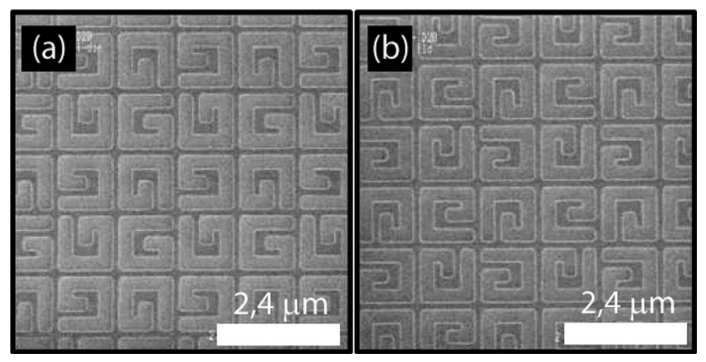

Fig. 1. Scanning electron microscopy pictures of the samples.

SHG-CD measurements were performed with a Mai-Tai femtosecond laser system at a wavelength of $800 \mathrm{~nm}$. The pulse length was approximately $100 \mathrm{fs}$ and the repetition rate was $80 \mathrm{MHz}$. Figure 2 shows the essential features of the experiment. The initial beam polarization was set by means of a Glan-Thompson polarizer. Subsequently, the rotation by an angle $\theta_{\mathrm{WP}}$ of a quar- 


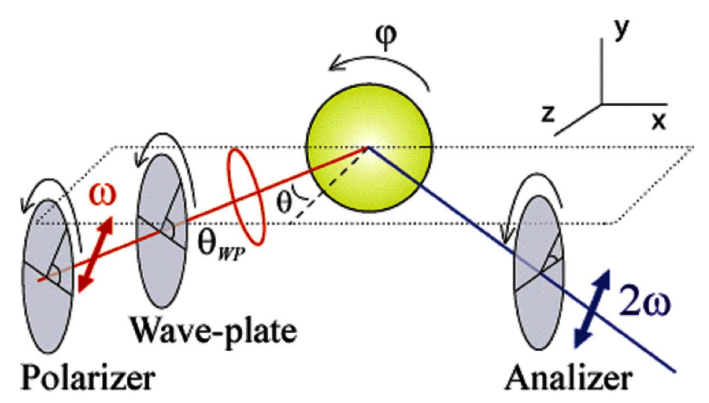

Fig. 2. Schematic diagram of the experimental configuration.

ter wave-plate modulated the polarization from left- to right-circularly polarized states. Next, the fundamental light, having a power of $300 \mathrm{~mW}$, was focused on the sample through a lens of $10 \mathrm{~cm}$ focal length. The angle of optical incidence on the sample $\theta$ was $45^{\circ}$. The sample was mounted on a motorized rotation stage. After reflection, the second harmonic was filtered through a BG39 filter, while the $800 \mathrm{~nm}$ radiation was blocked. The beam then passed through an analyzer and reached a XP2020 photomultiplier tube.

\section{Theoretical part}

The second-harmonic response can be described by a nonlinear polarization, which is expressed in the electric-dipole approximation as [3]:

$$
\boldsymbol{P}_{\mathrm{NL}}(2 \omega)=\chi^{(2)} \boldsymbol{E}(\omega) \boldsymbol{E}(\omega),
$$

where $\omega$ is the frequency of light, $\chi^{(2)}$ is the second order susceptibility tensor and $\boldsymbol{E}(\omega)$ is the electric field component of the incident light. It follows from this formula that the SHG can only be generated in non-centrosymmetric materials or regions of matter that lack inversion symmetry, such as surfaces and interfaces. $\chi^{(2)}$ is a third rank tensor and, depending on the symmetry of the material, the number of non-vanishing susceptibility components can be significantly reduced. For an in-plane isotropic chiral sample ( $C_{\infty}$ symmetry), the second order susceptibility becomes

$$
\chi^{(2)}=\left(\begin{array}{cccccc}
0 & 0 & 0 & \chi_{x y z} & \chi_{x x z} & 0 \\
0 & 0 & 0 & \chi_{y y z} & \chi_{y x z} & 0 \\
\chi_{z x x} & \chi_{z y y} & \chi_{z z z} & 0 & 0 & 0
\end{array}\right),
$$

where $\chi_{x z z}=\chi_{y z z}, \chi_{x x z}=\chi_{y y z}$ and $\chi_{x y z}=-\chi_{y x z}$. This last component leads to the appearance of nonlinear optical activity (the nonlinear equivalent of optical activity) and henceforth is responsible for the SHG-CD effect. This component is often referred to as the chiral one, since it is present only in chiral systems. The other components are referred to as achiral because they occur in both chiral and achiral systems.

It has been shown that the second-harmonic field can be conveniently written in terms of the components of the fundamental field $\boldsymbol{E}$ :

$$
\begin{aligned}
& I_{P-\text { out }}(2 \omega)=\mid f_{P-\text { out }} \boldsymbol{E}_{P-\text { in }}^{2}+g_{P-\text { out }} \boldsymbol{E}_{S-\text { in }}^{2} \\
& +\left.h_{P-\text { out }} \boldsymbol{E}_{P-\text { in }} \boldsymbol{E}_{S-\text { in }}\right|^{2},
\end{aligned}
$$

where the coefficients $f_{P \text {-out }}, g_{P \text {-out }}$ and $h_{P \text {-out }}$ are complex parameters, which are linear functions of the first-order nonlinear susceptibility tensor elements. In the following, we shall indicate the real and imaginary parts of these complex numbers by the indices 1 and 2 , respectively. The exact form of these coefficients depends on the symmetry of the sample. Furthermore, they are different for the reflected and transmitted second-harmonic light and they also depend on the angle of optical incidence.

After neglecting the refractive indices, for a chiral isotropic surface in the electric dipole approximation and in the reflection geometry, the dependence of $f_{P-\text { out }}$, $g_{P-\text { out }}$ and $h_{P-\text { out }}$ on the different susceptibility components takes the following form [4]:

$$
\begin{aligned}
& f_{P-\text { out }}=\sin \theta\left(2 \chi_{z z z} \sin ^{2} \theta+\chi_{z x x} \cos ^{2} \theta\right. \\
& \left.\quad+2 \chi_{x x z} \cos ^{2} \theta\right), \\
& g_{P-\text { out }}=\sin \theta\left(\chi_{z x x}\right), \\
& h_{P-\text { out }}=-\sin \theta\left(-2 \chi_{x y z} \cos \theta\right),
\end{aligned}
$$

where the subscripts $S$ and $P$ refer to the particular component of the SH-field.

Plotting the SHG intensity as a function of wave-plate angle allows one to extract the values of $f_{P-\text { out }}, g_{P-\text { out }}$ and $h_{P-\text { out }}$. For a wave-plate of retardation $\delta$ (where $\delta=\pi / 2$ is a half wave-plate and $\delta=\pi / 4$ is a quarter wave-plate), the fitting formula for the second-harmonic intensity as function of the polarization state of the incoming beam, which is determined by the initial state of linear polarization and the rotation of an angle $\theta_{\mathrm{WP}}$ of the wave-plate, can be expressed by the following formulae.

For $P$-polarized light before the quarter wave-plate [5]:

$$
\begin{aligned}
& I^{P-\mathrm{in}}(2 \omega)=\frac{1}{4}\left[-2 f_{1} \cos ^{2} \delta+\left(f_{1}+g_{1}\right) \sin ^{2} \delta\right. \\
& \quad+\left(f_{1}-g_{1}\right) \sin ^{2} \delta \cos 4 \theta_{\mathrm{WP}} \\
& \left.\quad-2 f_{2} \sin 2 \delta \cos 2 \theta_{\mathrm{WP}}+h_{1} \sin ^{2} \delta \sin \left(4 \theta_{\mathrm{WP}}\right)\right]^{2} \\
& \quad+\frac{1}{4}\left[-2 f_{2} \cos ^{2} \delta+\left(f_{2}+g_{2}\right) \sin ^{2} \delta\right. \\
& \quad+\left(f_{2}-g_{2}\right) \sin ^{2} \delta \cos 4 \theta_{\mathrm{WP}} \\
& \left.\quad+2 f_{1} \sin 2 \delta \cos 2 \theta_{\mathrm{WP}}+h_{1} \sin 2 \delta \sin 2 \theta_{\mathrm{WP}}\right]^{2}
\end{aligned}
$$

For $S$-polarized light before the wave-plate

$$
\begin{aligned}
& I^{S-\text { in }}(2 \omega)=\frac{1}{4}\left[2 g_{1} \cos ^{2} \delta-\left(f_{1}+g_{1}\right) \sin ^{2} \delta\right. \\
& +\left(f_{1}-g_{1}\right) \sin ^{2} \delta \cos 4 \theta_{\mathrm{WP}} \\
& \left.-g_{2} 2 \sin 2 \delta \cos 2 \theta_{\mathrm{WP}}+h_{1} \sin ^{2} \delta \sin \left(4 \theta_{\mathrm{WP}}\right)\right]^{2} \\
& +\frac{1}{4}\left[2 g_{2} \cos ^{2} \delta-\left(f_{2}+g_{2}\right) \sin ^{2} \delta\right.
\end{aligned}
$$




$$
\begin{aligned}
& +\left(f_{2}-g_{2}\right) \sin ^{2} \delta \cos 4 \theta_{\mathrm{WP}} \\
& \left.+g_{1} 2 \sin 2 \delta \cos 2 \theta_{\mathrm{WP}}-h_{1} \sin 2 \delta \sin 2 \theta_{\mathrm{WP}}\right]^{2}
\end{aligned}
$$

\section{Results}

Our samples have a 4-fold symmetry in the plane, which, in terms of the SHG is equivalent to an isotropic surface. However, anisotropy of the $\mathrm{Si} / \mathrm{SiO}_{2}$ substrate could contribute to an SHG-CD-like effect [6]. In order to eliminate this undesired signal, we have performed an absolute measurement of the SHG-CD in our samples. The procedure consisted of recording a SHG-CD pattern upon rotating the wave-plate $360^{\circ}$ in steps of $3^{\circ}$, for 36 steps of $10^{\circ}$ azimuthal rotation of the sample. Afterwards, all the azimuthal rotations were averaged to produce a single SHG-CD pattern, representative of a chiral isotropic surface. As it is well known, chirality is unaffected by rotation symmetry, while by this method any anisotropy is averaged out.

Figure 3 shows the SHG intensity versus the rotation angle of a quarter wave-plate at $800 \mathrm{~nm}$.

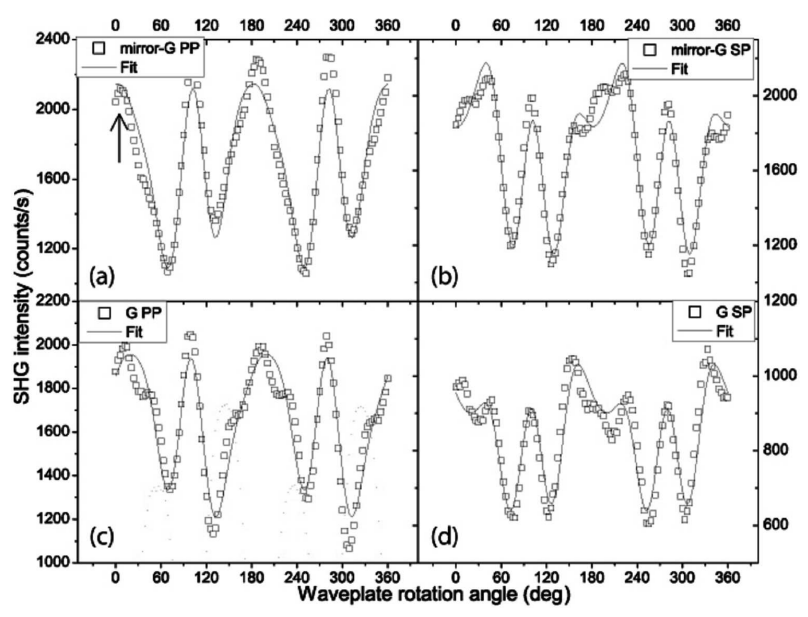

Fig. 3. The SHG intensity versus the rotation angle of a quarter wave-plate for two polarizer-analyzer combinations, i.e. $P-P$ in (a), (c) and $P-S$ and $S-P$ in $(b),(d)$. The data were recorded from the mirror-G sample in (a), (b) and from the G sample in (c), (d). The symbols indicate the experimental data and the lines are theoretical fits. The arrow in (a) indicates the position where the fast axis of the wave-plate is parallel to the incoming polarization.
The fits were performed by using 5 and 6 with a single set of fitting parameters, containing chiral $\left(h_{1, P-\text { out }}= \pm 1.75\right)$ and achiral isotropic $\left(f_{1, P-\text { out }}=\right.$ $-0.29, f_{2, P-\text { out }}=11.5, g_{1, P-\text { out }}=-8.2$ and $g_{2, P-\text { out }}=$ -1.6) contributions. Reversing the sign of the chiral fitting parameters fits the opposite handedness. The successful fitting procedure demonstrates that chiral nanostructures are very good model systems for the study of chirality with second harmonic generation.

\section{Conclusions}

We presented the SHG-CD measurements of G and mirror-G shaped gold nanostructures for $P$-polarized incoming light. By eliminating the contributions of anisotropy to the signal, an absolute measure of the SHG-CD was obtained. Samples with both enantiomorphs of the chiral pattern were prepared and, demonstrating the unambiguous presence of chirality, the SHG-CD response from two such samples was fitted simultaneously with a single set of fitting parameters.

\section{Acknowledgments}

We acknowledge financial support from the Fund for scientific research Flanders (FWO-V), the University of Leuven (GOA), Methusalem Funding by the Flemish government and the Belgian Inter-University Attraction Poles IAP Programmes. V.K.V., A.V.S. and W.G. are grateful for the support of FWO-Vlanders.

\section{References}

[1] T. Petralli-Mallow et al., J. Phys. Chem. 97, 1383 (1993).

[2] M. Kauranen et al., Adv. Mater. 7, 641 (1995).

[3] T. Verbiest, K. Clays, V. Rodriguez, Second-Order Nonlinear Optical Characterization Technique, CRC Press, Boca Raton 2009.

[4] M. Kauranen et al., J. Chem. Phys. 101, 8193 (1994).

[5] J.J. Maki et al., Phys. Rev. B 55, 5021 (1997).

[6] Xiaoqin Li et al., Appl. Phys. Lett. 89, 022102 (2006). 